\title{
Die Komfortzone verlassen und Verantwortung übernehmen
}

Professor Michael Lell ist Chefarzt für Radiologie und Nuklearmedizin im Klinikum Nürnberg. Im Interview spricht er über seine Erfahrungen als Radiologe im Nachtdienst und gibt jungen Kolleginnen/Kollegen Tipps, wie sie mit herausfordernden Situationen im Nachtdienst am besten umgehen.

Professor Lell, wie hoch ist der Anteil der Patientinnen/Patienten, die Sie wegen Verletzungen infolge von Schlägereien oder Unfällen nachts versorgen müssen?

Wir sehen in der Morgenbesprechung mehrere Fälle aus der Nacht, das sind - Verkehrsunfälle und Polytrauma-Patientinnen/-Patienten ausgenommen - meist Verletzungen des Mittelgesichts, des Nasenbeins, der Oribta oder des Schädels. Glücklicherweise sehen wir viel seltener Verletzungen der Halsweichteile und des Kehlkopfes. Die Ursachen reichen vom Sturz aus dem Bett über den Sturz im Badezimmer bis zu den Fällen häuslicher Gewalt oder Schlägereien in der Öffentlichkeit. Corona und die damit verbundenen Einschränkungen im öffentlichen Leben - sprich Ausgangssperre, Alkoholverbot im öffentlichen Raum, Ausfall von Konzerten, Sportveranstaltungen etc. - führten zu einem deutli- chen Rückgang. Für CT-Untersuchungen des Mittelgesichts im Nachtdienst liegt der Rückgang bei uns bei etwa $35 \%$.

Was sind das für Menschen, die nachts verletzt in Ihre Klinik kommen?

Das ist eine bunte Mischung. Bei den SturzPatientinnen/-Patienten sind es natürlich vorwiegend ältere Menschen. Bei dieser Gruppe hat aus naheliegenden Gründen auch Corona keinen Einfluss auf die Frequenz. Bei den tätlichen Auseinandersetzungen sehen wir ein breites Altersspektrum, das von Jugendlichen bis ins höhere Erwachsenenalter reicht. Bei denen ist oft das Management der Betroffenen, die nicht selten mit ihren Angehörigen oder Freundinnen/Freunden ankommen, schwieriger als die eigentliche radiologische Leistung. Gerade die MTRA, die den unmittelbaren Kontakt zu den teils aggressiv auftretenden Patientinnen/Patienten oder Angehörigen haben, stehen dabei im Fokus. Leider ist in den letzten Jahren ein sehr unschöner Trend zu Aggression gegenüber Mitarbeiterinnen/Mitarbeitern festzustellen. Mit der Einführung von Sicherheitspersonal, gezielten Deeskalationsschulungen, baulichen Veränderungen etc. sind gezielte Schritte zur Verbesserung der Sicherheit der

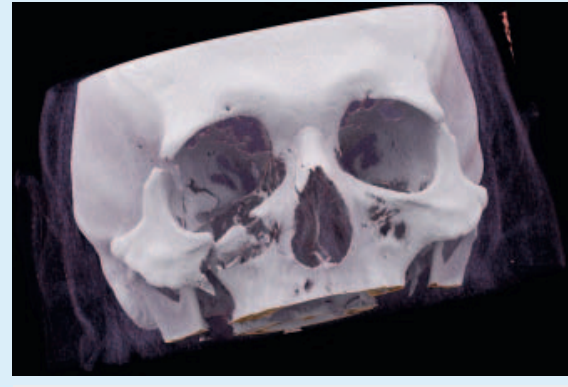

Tripod-Fraktur eines Gesichtsschädels OKlinikum Nürnberg

Mitarbeiterinnen/Mitarbeiter eingeleitet worden.

Wie sieht die radiologische Versorgung dieser Verletzten aus?

Häufig ist das eine Kombination aus Ultraschalluntersuchung und einer CT-Untersuchung des Mittelgesichts, des Schädels, der Wirbelsäule oder des Halses. Je nach Trauma auch des Thorax oder des Abdomens.

Bei der radiologischen Versorgung von Verletzten spielt sicherlich auch der Faktor Zeit eine Rolle. Wie bestimmt dieser Faktor Ihre Arbeit als Radiologe? 
Zumindest in den größeren Häusern ist das so geregelt, dass eine flächendeckende Sofortbefundung umgesetzt ist. Das bedeutet, dass jede Aufnahme unmittelbar nach der Erstellung radiologisch befundet wird. Gewisse zeitliche Verzögerungen gibt es immer dann, wenn die Radiologin/der Radiologe gerade mit einer Ultraschalluntersuchung oder einem CT beschäftigt ist.

Die Versorgung Verletzter ist auch eine interdisziplinäre Arbeit. Mit welchen medizinischen Fachgebieten arbeitet die Radiologie besonders intensiv zusammen?

Im Nachtdienst ist das in erster Linie das Team der Notaufnahme. Je besser die Kommunikation zwischen den beiden Abteilungen funktioniert, umso schneller und sicherer gelingt die Beurteilung der Untersuchungen. Der wichtigste Punkt aber ist der direkte Kontakt der Radiologinnen/Radiologen mit den Patientinnen/Patienten! Der Augenschein, eine kurze eigene Anamnese oder Untersuchung klären sehr viele Fragen.

Inwieweit ist die Radiologie in die medizinische Nachsorge eingebunden?
In der Folge sehen wir die Patientinnen/ Patienten kaum mehr, da in aller Regel die weitere Behandlung ambulant abläuft.

Vor dem Hintergrund Ihrer ärztlichen Erfahrungen: Was sind die größten Herausforderungen für Radiologinnen/Radiologen im Nachtdienst?

In den ersten Nachtdiensten sieht auch das Gewohnte plötzlich ungewohnt aus und es fehlt das unmittelbare Feedback einer erfahrenen Radiologin/eines erfahrenen Radiologen. Dann kommt man schon mal ins Grübeln: „Ist das jetzt eine Fraktur oder doch nur ein Gefäßkanal oder eine Sutur?" Die Entscheidung, die man fällt, hat ja in der Regel eine unmittelbare Auswirkung auf die Therapie. Das bedeutet, man verlässt seine Komfortzone und muss Verantwortung übernehmen. Aber die Aufregung legt sich bald und man wird immer routinierter. Ein anderes Feld, auf dem man sich im Dienst weiterentwickeln muss, ist das eigene Zeitmanagement. Zusätzlich zu der primären Aufgabe der Diagnostik kommen Telefonate von Kolleginnen/Kollegen auf den Stationen, der Notaufnahme etc., die alle etwas von einem haben oder wissen wollen. Der Ein- satz im Schockraum und besonders das "Schockraum-CT“ ist ein besonderer Stressfaktor. Außerdem gibt es noch das bereits angesprochene Management der Alkoholisierten oder Intoxikierten.

Was würden Sie Kolleginnen/Kollegen mit auf den Weg geben, die zum ersten Mal im Nachtdienst arbeiten?

Ruhe bewahren, sich auf die aktuell zu behandelnde Person fokussieren, auf seine Fähigkeiten vertrauen und gerade am Anfang der Diensttätigkeit nicht davor zurückschrecken, sich Rat zu holen. Wir haben im Klinikum Nürnberg ein System mit Zwischendiensten entwickelt, bei dem die jungen Kolleginnen/Kollegen langsam und stufenweise in die Dienste eingeführt werden. Darüber hinaus gibt es bei uns den Vorteil, dass mit den 2 Standorten immer ein weiterer diensthabender Radiologe/eine weitere diensthabende Radiologin vor Ort ist. Der Hintergrunddienst ist selbstverständlich immer telefonisch erreichbar und hat die Möglichkeit, über einen gesicherten $\mathrm{Zu}$ gang von zu Hause aus auf das PACS zuzugreifen. 